\title{
Blind Deconvolution of Widefield Fluorescence Microscopic Data by Regularization of the Optical Transfer Function (OTF)
}

\author{
Margret Keuper ${ }^{1}$, Thorsten Schmidt ${ }^{1}$, \\ Maja Temerinac-Ott ${ }^{1}$, Jan Padeken ${ }^{2}$, Patrick Heun ${ }^{2}$, Olaf Ronneberger ${ }^{1}$, Thomas Brox ${ }^{1}$ \\ ${ }^{1}$ Department of Computer Science, University of Freiburg, and \\ BIOSS, Centre for Biological Signalling Studies \\ ${ }^{2}$ Max Planck Institute of Immunobiology and Epigenetics, Freiburg \\ keuper@cs.uni-freiburg.de
}

\begin{abstract}
With volumetric data from widefield fluorescence microscopy, many emerging questions in biological and biomedical research are being investigated. Data can be recorded with high temporal resolution while the specimen is only exposed to a low amount of phototoxicity. These advantages come at the cost of strong recording blur caused by the infinitely extended point spread function (PSF). For widefield microscopy, its magnitude only decays with the square of the distance to the focal point and consists of an airy bessel pattern which is intricate to describe in the spatial domain. However, the Fourier transform of the incoherent PSF (denoted as Optical Transfer Function (OTF)) is well localized and smooth. In this paper, we present a blind deconvolution method that improves results of state-of-theart deconvolution methods on widefield data by exploiting the properties of the widefield OTF.
\end{abstract}

\section{Introduction}

To analyze living cells, widefield fluorescence microscopy plays an important role, because it is prevalently available and, compared to confocal microscopy, has advantages concerning temporal resolution and phototoxicity. In contrast to confocal laser scanning microscopy, where the sample is scanned point by point, the 3D information of the specimen is recorded in a stack of $2 \mathrm{D}$ images. Since the whole specimen is illuminated for every image, light is always recorded from in-focus and from out-of-focus planes. Thus, the resolution along the optical axis is very limited [23]. The recorded out-of-focus light also determines the impulse response, the point-spread function (PSF) of the imaging system. The image formation of fluorescence microscopes can be approximated by a convolution of the original specimen function $s$ with the PSF $h$ and a voxelwise

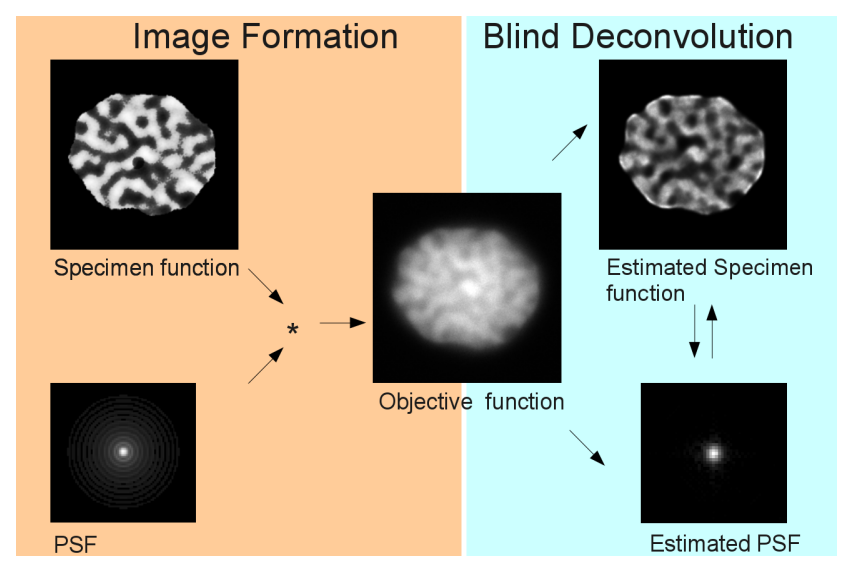

Figure 1. As for widefield microscopy the convolution of the signal with the PSF makes the data hard to process, the aim is to correct for the effect of the PSF by a deconvolution technique.

noise function $n: \mathbb{R}^{+} \rightarrow \mathbb{R}^{+}$as given in the following equation (see figure 1)

$$
o=n(s * h) .
$$

The objective function $o$ is the recorded image data. As for widefield microscopy the convolution of the signal with the PSF makes the data hard to process, the aim is to correct for the effect of the PSF by a deconvolution technique. In principle, the PSF can be determined for each combination of objective, filters and light path in a specific microscope by a calibration procedure. However, it depends on many parameters, is subjected to changes in the recording system like thermal expansions, aberrations, or the optical properties of the recorded specimen. Therefore, it is beneficial to use the calibration outcome only as an initial estimate for blind image deconvolution, where the PSF $h$ is refined while estimating the specimen $s$ in a joint optimization procedure. 
Deconvolution of 3D data from widefield microscopy is particularly hard because a part of the information is lost in the recording even if the perfect PSF was given [23]. Due to the so-called missing cone of the widefield recording system, certain spatial frequencies simply can not be captured. Along the optical axis, even low frequency information is lost.

\subsection{Related Work}

Image deconvolution in general is a vast topic with applications in the fields of microscopic image reconstruction, motion deblurring, or the deblurring of astronomic images. The algorithms depend on the assumptions on the noise function $n$. We refer to [23] for a survey on several deconvolution methods in widefield microscopy. In [3] several nonlinear deconvolution methods as the LucyRichardson method [17, 22], the Maximum Likelihood Expectation Maximization (MLEM) method [26], and a maximum entropy method [19] are described in detail. A stateof-the-art motion deblurring method for natural images is given for example in [12].

For the deconvolution of widefield microscopic data, MLEM deconvolution methods have proven to be very efficient $[23,10,4,14]$. However, they require regularization of both the specimen function and the deconvolution kernel. Otherwise they do not converge and tend to amplify noise and to introduce ringing artifacts [23, 25]. Common methods use prior models on the specimen function such as the Tikhonov-Miller penalizer [30] or Total Variation (TV) regularization [5], enforcing smoothness in the deconvolved data and thus reducing noise. In [4], an intensity penalization for the data term is used, hindering the data from taking too high values.

For the regularization of the reconstructed PSF, usually some specific prior knowledge on the image formation is used. In $[18,14]$ the regularization is done by a projection of the current estimate onto a given PSF model. In [18], the parameters of the PSF model (given in [6]) are estimated in each step. In [14], the current estimate is projected onto the space spanned by all simulated PSFs [29] using a kernel PCA. With this method, different PSFs with and without spherical aberrations can be continuously modeled. However, there is no guarantee that the reconstructed kernel is actually a valid PSF.

In [11], an adaptive image deconvolution algorithm (AIDA) is presented: a blind deconvolution framework for $2 \mathrm{D}$ and 3D data. The basic assumption is that the PSF of the optical system is approximately known and either given in the form of the optical transfer function (OTF) or as several OTFs (computed for example from given PSF simulations), of which the true OTF is expected to be a linear combination. During the deconvolution process, the PSF estimation

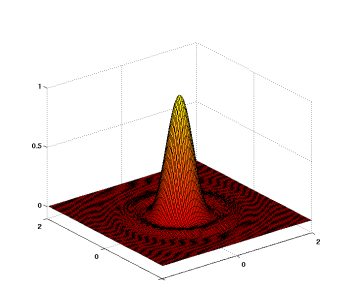

(a) Airy pattern

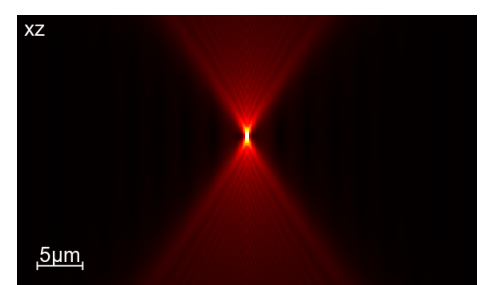

(b) Simulated PSF (xz-section) using [16, 6].

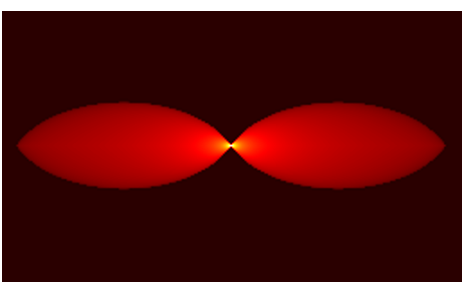

(c) Simulated OTF (kx-kz-section) using [21].
Figure 2. PSF and OTF simulations. While the PSF is not smooth and has an infinitely large support, the OTF has a limited support and varies smoothly.

is bound to these given OTFs by the $L_{2}$-norm. Also the data fidelity term in [11] is based on the $L_{2}$-norm and in contrast to MLEM does not assume Poisson statistics. As [14], [20] use the PSF model of [29] in the blind MLEM deconvolution framework. Similarly to [11], they bind the estimated PSF with a quadratic term to the simulated PSF.

All the before mentioned deconvolution methods describe the PSF in the spatial domain. This has major disadvantages in the case of widefield microscopy: the widefield PSF has an infinitely large support in the spatial domain and is not smooth (compare figure 2), such that discrete sampling of the PSF in the spatial domain leads to artifacts. In this paper, we present a regularization method of the deconvolution kernel, that is based on imposing constraints on the PSF in the frequency domain, where it is easy to describe and well localized. We thus propose to use a regularization of the Fourier transform of the deconvolution kernel, employing its frequency domain properties.

\section{Physical Model}

The PSF of a fluorescence microscope depends on several parameters of the recording system such as the Numerical Aperture, the emission wavelength $\lambda$, and the position of the recorded object. The Numerical Aperture is defined as $\mathrm{NA}=n_{\text {imm }} \cdot \sin \Theta$, where $n_{\text {imm }}$ is the refractive index of the immersion medium, and $2 \Theta$ is the angular aperture of the objective. Most importantly, the finite lens aperture introduces diffraction ring patterns in the recorded xy-sections [23], the so called Airy pattern (see figure 2(a)), that limits the resolution of the recording system. Because of the Airy pattern, the PSF of widefield microscopes does not have a compact support $[6,1]$ and usually has values of an unnegligible range in the whole image domain $\Omega$. There are several 
methods to theoretically model the recording system and thus model the PSF, e.g. [29, 6, 1] or its Fourier domain equivalent, the optical transfer function (OTF) [27, 21, 24]. A theoretical PSF according to [6] computed with [16] is depicted in figure 2(b).

While other deconvolution methods focus on modeling the PSF, we show the advantages of working with the OTF. The OTF directly describes the properties of an imaging system. Frequencies outside the OTF support are cut off during the imaging process. The OTF support of a widefield microscope is depicted in figure 3. The recorded light field is assumed to be nearly monochromatic with constant wavelength $\lambda$. The corresponding wave-number is $k=2 \pi / \lambda$ for the whole field, which means that the Fourier transform of the field amplitude has non-zero values only on a shell with radius $2 \pi / \lambda$ (figure $3(\mathrm{a})$ ). Since only the light from a limited angle $\Theta$ can be recorded (figure 3(b)), the recorded information is limited to a "cap" of the spherical shell in the Fourier space (figure 3(c)). The autocorrelation of this spherical cap is the region from which intensity information is accessible, i.e. the support of the OTF (figure 3(d)). Compare $[9,21]$ for more detailed information.

The OTF is rotation symmetric around the $\mathrm{k}_{\mathrm{z}}$ frequency axis. An algorithm for computing the OTF values for nonaberrated optical systems is given in [21]. An example OTF is shown in figure 2(c).

It can be observed that in $\mathrm{k}_{\mathrm{z}}$-direction, the OTF drops to zero very quickly. The conical region where the OTF is zero is called missing cone and explains the significant amount of blur in z-direction. Here, even low frequency information is cut off. Inside its support, the OTF varies smoothly, allowing for a regularization using a total variation (TV) prior.

\section{Blind Maximum Likelihood Expectation Maximization Deconvolution}

In the following, we briefly sketch the blind Maximum Likelihood Expectation Maximization Deconvolution (MLEM) algorithm. A more detailed derivation is given in [3]. The aim of this algorithm is to find the specimen function estimate $\hat{s}$ and point spread function estimate $\hat{h}$ that maximize the conditional probability of the measured objective function:

$$
\hat{s}, \hat{h}=\arg \max _{s, h}\{p(o \mid s, h)\},
$$

where $o$ denotes the objective function, $s$ the specimen function, and $h$ the PSF from equation (1). Since photon noise is Poisson distributed [2], the recorded objective function $o(\mathbf{x})$ at voxel $\mathbf{x}$ can be viewed as a sample of a Poisson distribution with mean $(s * h)(\mathbf{x})$. Since the intensity at each voxel in $o$ is drawn from an independent Poisson process, the objective function $o$ given the specimen $s$ and the PSF

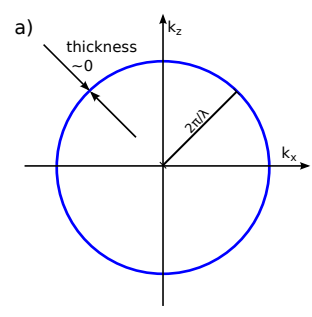

b)
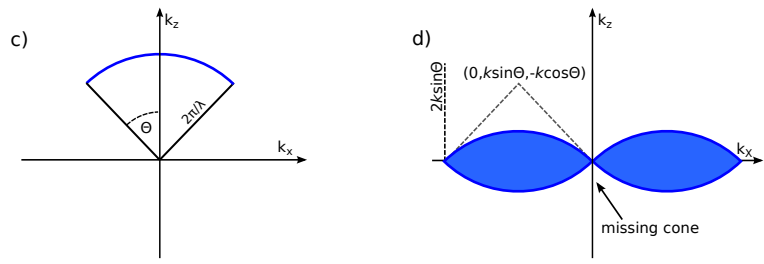

Figure 3. Illustration of the support of the widefield OTF [9, 21]. (a) The spherical shell on which the Fourier transform of the monochromatic light field amplitudes is non-zero. (b) The objective lens with a finite acceptance angle $\Theta$. (c) The spherical cap containing the recorded amplitude information. (d) The resulting OTF support. The conical area in $k_{z}$ direction, where even low frequency information is lost, is called the missing cone.

$h$ can be expressed in an ML way as the product

$$
p(o \mid s, h)=\prod_{\mathbf{x} \in \Omega \subseteq \mathbb{R}^{3}} \frac{((s * h)(\mathbf{x}))^{o(\mathbf{x})} \exp (-(s * h)(\mathbf{x}))}{o(\mathbf{x}) !},
$$

where $\Omega \subseteq \mathbb{R}^{3}$ is the recording domain. Instead of maximizing (3), we minimize the negative log-likelihood function

$$
J_{\text {MLEM }}(s, h)=\int_{\Omega} s * h-o \cdot \log (s * h)+\log (o !) d \mathbf{x} .
$$

The minimization is done by differentiating (4) with respect to $s$ and $h$ and set the derivative to zero. The resulting iterative, multiplicative scheme is given by alternating:

$$
\begin{array}{r}
\hat{s}_{k+1}(\mathbf{x})=\hat{s}_{k}(\mathbf{x}) \cdot\left(h^{m} * \frac{o}{\left(h * \hat{s}_{k}\right)}\right)(\mathbf{x}) \quad \text { and } \\
\hat{h}_{k+1}(\mathbf{x})=\frac{\hat{h}_{k}(\mathbf{x})}{\int_{\Omega} \hat{s}_{k}(\mathbf{y}) d \mathbf{y}} \cdot\left(\hat{s}_{k}^{m} * \frac{o}{\left(\hat{h}_{k} * \hat{s}_{k}\right)}\right)(\mathbf{x}),
\end{array}
$$

where $h^{m}(\mathbf{x})=h(-\mathbf{x})$ is the mirrored PSF, $s^{m}(\mathbf{x})=$ $s(-\mathbf{x})$ denotes the mirrored specimen function, and the PSF $h$ is assumed to integrate to one: $\int_{\Omega} h^{m}(\mathbf{x}) d \mathbf{x}=1$.

This update scheme, which is for the specimen function update equivalent to the Lucy-Richardson deconvolution, has some desired properties such as conservation of the positivity of the radiant flux [3]. To avoid numerical problems when the denominator becomes zero, we set $\hat{s}_{k+1}(\mathbf{x})=0$ if $\left(h * \hat{s}_{k}\right)(\mathbf{x})=0$. 


\subsection{Residual Denoising}

The effect of noise can be reduced by denoising the residual $R_{k}$ derived as

$$
o=\hat{s}_{k} * \hat{h}_{k}+R_{k} \quad \leftrightarrow \quad R_{k}=o-\hat{s}_{k} * \hat{h}_{k}
$$

Since the function $h$ is considered a blurring kernel and $\hat{h}_{k}$ is an approximation of $h, \hat{s}_{k} * \hat{h}_{k}$ should be noise-free such that all the noise of $o$ is contained in $R_{k}$ [14]. $R_{k}$ can be denoised with any denoising function, such as wavelet denoising [28] or median filtering [14]. We follow [14] and compute the denoised residual $\bar{R}_{k}$ using a $3 \times 3 \times 3$ median filter. This allows for the computation of the residual denoised objective function $\bar{o}=\hat{s}_{k} * \hat{h}_{k}+\bar{R}_{k}$, which replaces $o$ in the update scheme (5) and (6).

\subsection{Regularization}

In the blind deconvolution setting, the above ML formulation is ill-posed. Regularization must be added, leading to a maximum a-posteriori formulation known as penalized MLEM [7, 8]

$$
\hat{s}, \hat{h}=\arg \max _{s, h}\{p(s, h \mid o)\} .
$$

The penalized MLEM functional is given by [13]

$$
J_{\text {PMLEM }}(s, h)=J_{\text {MLEM }}(s, h)+\lambda_{s} P_{s}(s)+\lambda_{h} P_{h}(h) .
$$

where $p(s)=\exp \left(-\lambda_{s} P_{s}(s)\right)$ and $p(h)=$ $\exp \left(-\lambda_{h} P_{h}(h)\right)$ are general prior probability functions for the specimen function and the deconvolution kernel respectively. $P_{s}(s)$ and $P_{h}(h)$ can be arbitrary penalty functionals, returning high values for inputs that do not match the prior knowledge and low values for inputs that do. $\lambda_{s}$ and $\lambda_{h}$ are positive constants, that serve as weights for the penalty terms. Usually, the optimization is implemented using Greens multiplicative one step late algorithm [8], yielding the update

$$
\hat{s}_{k+1}(\mathbf{x})=\frac{\hat{s}_{k}(\mathbf{x}) \cdot\left(\hat{h}_{k}^{m} * \frac{o}{\left(\hat{h}_{k} * \hat{s}_{k}\right)}\right)(\mathbf{x})}{1+\lambda_{s}\left(\frac{\partial}{\partial s} P_{s}\right)\left(\hat{s}_{k}(\mathbf{x})\right)}
$$

and

$$
\hat{h}_{k+1}(\mathbf{x})=\frac{\hat{h}_{k}(\mathbf{x}) \cdot\left(\hat{s}_{k}^{m} * \frac{o}{\left(\hat{h}_{k} * \hat{s}_{k}\right)}\right)(\mathbf{x})}{\int_{\Omega} \hat{s}_{k}(\mathbf{y}) d \mathbf{y}+\lambda_{h}\left(\frac{\partial}{\partial h} P_{h}\right)\left(\hat{h}_{k}(\mathbf{x})\right)} .
$$

Many statistical priors for the specimen function have been investigated in literature, from simple intensity regularizations to more elaborate priors as the Gaussian prior or the entropy prior $[4,31,30]$. The total variation regularization [5] has the desired property of preserving the edges in the data while suppressing noise and oscillations and is therefore most often employed. For the TV regularization, $P_{s}(s)=\int_{\Omega}\|\nabla s(\mathbf{x})\| d \mathbf{x}$ and the derivative is given by $\left(\frac{\partial}{\partial s} P_{s}\right)\left(\hat{s}_{k}(\mathbf{x})\right)=-\operatorname{div}\left(\frac{\nabla \hat{s}_{k}(\mathbf{x})}{\left\|\nabla \hat{s}_{k}(\mathbf{x})\right\|}\right)$, where $\operatorname{div}(s)=$ $\frac{\partial s}{\partial x_{1}}+\frac{\partial s}{\partial x_{2}}+\frac{\partial s}{\partial x_{3}}$ is the divergence.

\subsection{TV Regularization in the Frequency Domain}

For widefield recordings, we know that the PSF is not smooth, such that a regularization of the kernel by imposing smoothness is not reasonable. In contrast, we have seen in section 2 that the Fourier transform of the PSF, the OTF $\mathcal{F}(h)$, has a well defined support region and is smooth inside this region.

To develop a regularizer that acts on the OTF, we decompose the OTF into amplitude and phase:

$$
\mathcal{F}(h)=\underbrace{|\mathcal{F}(h)|}_{\text {amplitude }} \cdot \underbrace{\frac{\mathcal{F}(h)}{|\mathcal{F}(h)|}}_{e^{2 \phi}} .
$$

and penalize the variation of the amplitude:

$$
P_{h}(h)=\int\|\nabla|\mathcal{F}(h)|(\xi)\| d \xi .
$$

The optimum of this TV energy, a constant amplitude of the OTF, does not account for the fact that the support of the OTF is limited. We therefore enforce a limited support by shrinking all values outside the support region to zero [10]. To make sure that valid frequencies are not cut off, we compute the largest theoretically possible OTF support for a widefield microscope according to [21]. The OTF is largest for the smallest possible emission wavelength and the largest possible $\sin (\Theta)$ where $\Theta$ is the angle of the maximum cone of light that can enter the objective lens (see section 2, figure 3). We thus compute the maximal OTFsupport with wavelength $\lambda=380 \mathrm{~nm}$ and $\Theta=\frac{\pi}{2}-\epsilon$; see figure 4 . Values outside this support are set to zero in every iteration of the deconvolution update. This operation can introduce negative values in the current PSF estimate. In order to reestablish the non-negativity of the PSF estimate, [10] proposed to cut off negative PSF values and set them to zero. This non-linear operation caused numerical problems in our data. It reintroduces frequencies outside the OTF support. Additionally, PSF values that are cut off can not take on any value different from zero in the multiplicative update scheme. Instead of setting negative values to zero, we normalize the PSF to a valid range to avoid these problems. For minimizing the resulting functional with TV regularization of the kernel in the frequency domain (KFTV), we compute the gradient with the calculus of variations. Since 


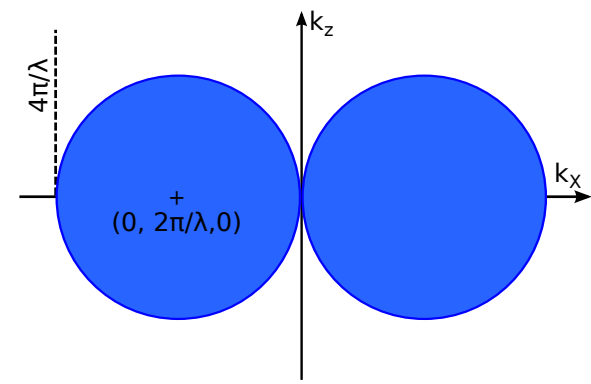

Figure 4. For $\Theta \rightarrow \frac{\pi}{2}$, the OTF support approaches a horn torus with radius $\frac{2 \pi}{\lambda}$.

the Fourier transform is linear, a variation in the spatial domain can be written in the Fourier domain as

$$
\int\|\nabla|\mathcal{F}(h+\epsilon g)|(\xi)\| d \xi=\int\|\nabla|\mathcal{F}(h)+\epsilon \mathcal{F}(g)|(\xi)\| d \xi .
$$

It results in

$$
\begin{aligned}
\left(\frac{\partial}{\partial h} P_{h}\right) & \left(\hat{h}_{k}(\mathbf{x})\right)= \\
& -\mathcal{F}^{-1}\left(\operatorname{div}\left(\frac{\nabla\left|\mathcal{F}\left(\hat{h}_{k}\right)\right|}{\left\|\nabla\left|\mathcal{F}\left(\hat{h}_{k}\right)\right|\right\|}\right) \cdot \frac{\mathcal{F}\left(\hat{h}_{k}\right)}{\left|\mathcal{F}\left(\hat{h}_{k}\right)\right|}\right)(\mathbf{x}),
\end{aligned}
$$

that can be used in the multiplicative update for the deconvolution kernel (11). For the detailed derivation of the gradient, we refer to the supplemental material.

Implementation For efficiency reasons, we implement the convolutions using the Fast Fourier transform ${ }^{1}$. Since the OTF has a limited support, the true PSF does not. In practice, only a finite array is available to represent the PSF. If this array is too small, the reconstructed PSF can have non-zero values at the array boundaries such that zeropadding for the convolutions introduces artifacts. In our implementation, we initially extend the array by padding it with zeros, assuming that the initial PSF estimate drops to zero at the array boundaries. During the deconvolution process, we update the PSF in the whole, extended array, even though we know that this might again lead to aliasing effects after applying a circular convolution, if the PSF grows too large. With this implementation, we did not encounter any problems.

\section{Experiments and Results}

We conducted experiments on synthetic data as well as on real microscopic recordings. All results are reported in terms of the root mean squared error (RMSE)

$$
\operatorname{RMSE}=\left(\frac{1}{N} \sum_{i=1}^{N}(d(i)-c \cdot \hat{d}(i))^{2}\right)^{\frac{1}{2}}
$$

\footnotetext{
${ }^{1}$ We use the FFTW implementation from http://www. fftw.org.
}

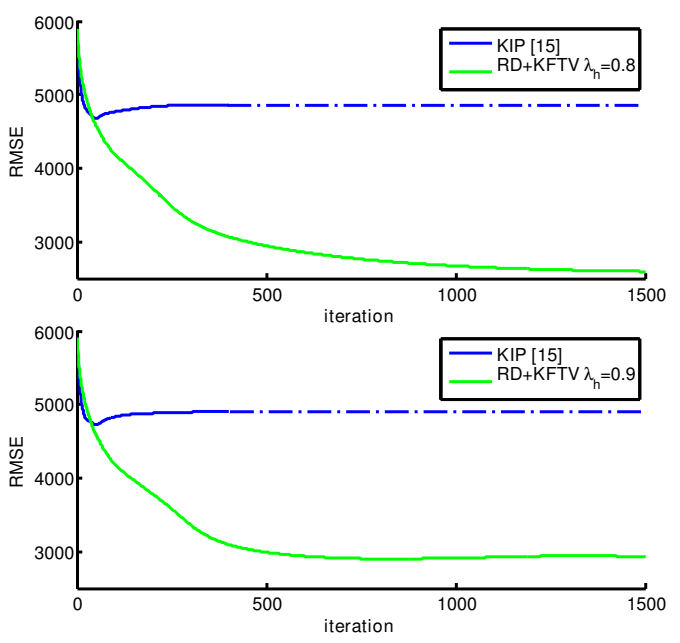

Figure 5. Development of the RMSE during the iterations on the synthetic data sim.1 (top) and sim.2 (bottom). KIP [15] converges already after 400 iterations and the dash-dotted line indicates its RMSE value at convergence. With KIP, the RMSE increases again after some iterations while the KFTV model converges to the solution with smallest RMSE.

where $\hat{d}$ is the final estimate for the reconstructed data $d$ and

$$
c=\frac{\sum_{i=1}^{N} d(i)}{\sum_{i=1}^{N} \hat{d}(i)}
$$

The normalization by $c$ accounts for different scalings of the data and has previously been used for the evaluation of state-of-the-art deconvolution methods (e.g. in [14]).

\subsection{Synthetic Data}

In a first experiment we compared to the KIP method from [15] on their dataset ${ }^{2}$. It consists of two widefield microscopic recordings with different levels of Poisson noise of a synthetic HeLa cell nucleus (sim.1 with SNR=1.63 and sim. 2 with $\mathrm{SNR}=1.61$ in figure 6). KIP [15] uses a simple intensity regularization on the reconstructed PSF preventing the reconstructed kernel from collapsing. Figure 6 shows results for the baseline method KIP computed for the parameter $\lambda_{\mathrm{KIP}}=35$, an OTF masking without TV regularization (OTF mask), and our method KFTV in two orthogonal views. Residual denoising (RD) has been used to regularize the specimen function. As expected, a kernel regularization in the frequency domain has clear advantages over the simple KIP [15] baseline, both visually and in terms of RMSE. While the resulting RMSE with OTF mask and KFTV is quite similar for sim.1, KFTV brings a significant improvement on the noisier data sim.2. The development of the RMSE of the reconstructed specimen function over

\footnotetext{
${ }^{2}$ The dataset can be downloaded from lmb.informatik uni-freiburg.de/people/keuper/data
} 


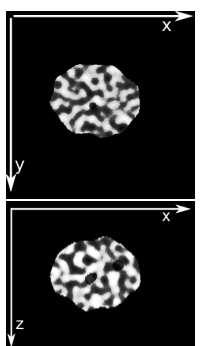

(a) Ground Truth

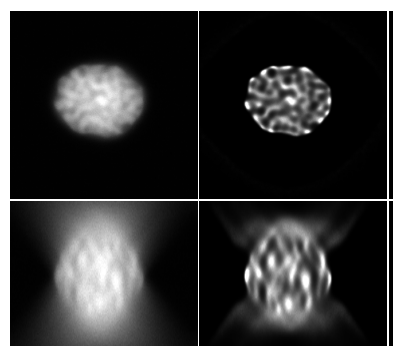

(b) $\operatorname{sim} .1$

RMSE $=5897.6$

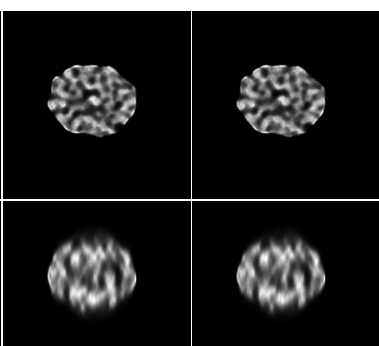

(d) RD+

OTF mask

RMSE $=2596.3$ (e) RD+KFTV

$\lambda_{h}=0.8$

RMSE $=2596.2$

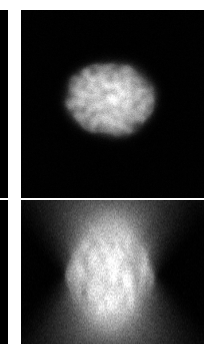

(f) $\operatorname{sim} .2$

RMSE $=5907.4$

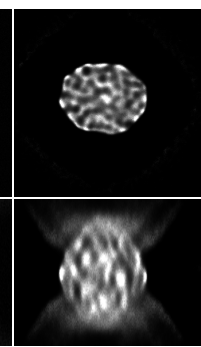

(g) KIP [15] RMSE $=4908.1$

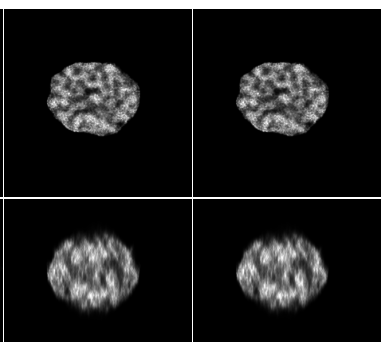

(h) RD+

OTF mask

RMSE $=3013.2$ (i) RD+KFTV

$\lambda_{h}=0.9$

RMSE $=2925.5$

Figure 6. KFTV blind deconvolution results on the synthetic datasets sim.1 (SNR=1.63) and sim.2 (SNR=1.61) displayed in the central xy-section (top row) and the central xz-section (bottom row). The specimen function has been regularized using RD. The reduced blur in z-direction obtained by OTF mask and KFTV shows the advantage of constraining the kernel function in the frequency domain.

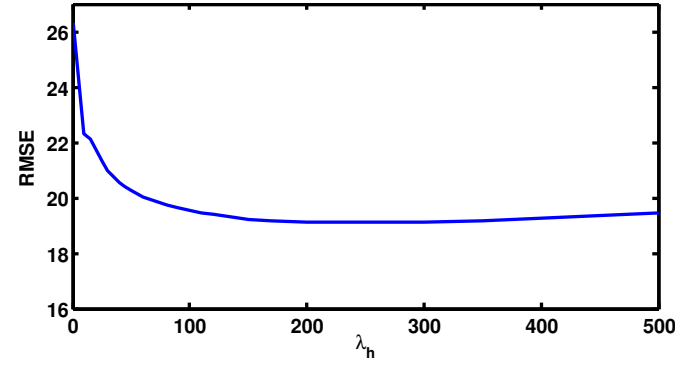

Figure 7. RMSE depending on the regularization parameter $\lambda_{h}$ for RD+KFTV on the torus data set [14] (compare figure 8(b)). The method is stable for a large parameter range. However, OTF mask $\left(\lambda_{h}=0\right)$ performs signifcantly worse than higher $\lambda_{h}$ weightings.

the iterations is plotted in figure 5. With KIP [15], we observe that the RMSE increases again after some iterations even though the energy $J_{\text {MLEM }}$ converges. In [23], this undesired property has been described as a common effect in MLEM-deconvolution. It indicates that the model is a poor approximation of the actual problem. In contrast, our model based on OTF regularization reaches its optimum close to the solution with the smallest RMSE.

Comparison to Methods with complex State-of-the-Art PSF Models In a second experiment we compare to the sophisticated learning based method from [14] on their test data. We further show the results using the method from [11] on this same data. ${ }^{3}$. [14] perform blind MLEM deconvolution with RD in the specimen function and in the kernel update. In their work, the space of possible kernel functions is learned a priori. It is spanned using kernel PCA computed on a synthetically generated library of PSFs. In every iteration, the current kernel estimate is projected onto this space. The synthetic datasets used in [14] are $129 \times 129 \times 129$ voxel volumes with a voxel size of $0.1 \mu \mathrm{m}^{3}$ that are blurred

\footnotetext{
${ }^{3}$ Thanks to Tal Kenig who kindly let us use his data for evaluation.
}

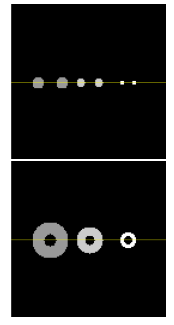

(a) Ground truth

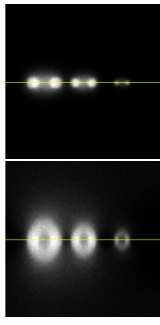

(b) Blurred
noisy data. RMSE $=35.81$

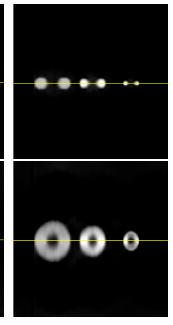

(c) AIDA [11]

RMSE $=25.47$

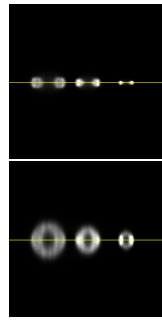

(d) kernel PCA (e) RD+KFTV [14] $\quad \lambda_{h}=200$ RMSE $=19.30 \quad$ RMSE $=19.10$
Figure 8. Toy data used in [14] and the deconvolution results with the different methods AIDA [11], kernel PCA [14], and our proposed method KFTV. Two orthogonal views are displayed: the xy-view (top row) and the xz-view (bottom row). KFTV provides superior results without system specific PSF models. Sub-figures (a) - (d), and the respective RMSE values are taken from [14].

with synthetically generated PSFs and corrupted by Poisson noise. All synthetic PSFs are generated using the method from [29]. The initialization is done using a symmetric PSF support computed according to [13]. For the comparison to [14] and [11], we also use RD for the specimen function and the kernel update. Our initialization is done as in [14] with the symmetric PSF support. The results after 400 iterations are displayed in figure 8 . For the highly asymmetric data, where the PSF is only initialized with its support, we chose a high KFTV weighting with $\lambda_{h}=200$. In figure 7, the development of the RMSE with respect to the chosen weighting is displayed. In a qualitative evaluation as well as in terms of RMSE, our method shows convincing results on the dataset.

\subsection{Drosophila S2 Cell Recordings}

Real widefield microscopic recordings were taken from fixed samples of DAPI stained Drosophila S2 cell nuclei. For the widefield microscope, a voxel size of $0.0642 \times$ $0.0642 \times 0.2 \mu \mathrm{m}^{3}$ was used. With the same settings, a 

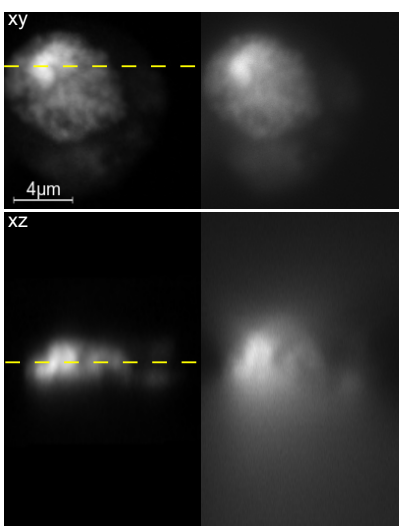

(a) Spinning Disk (b) Widefield (Ground Truth)

RMSE $=21.25$

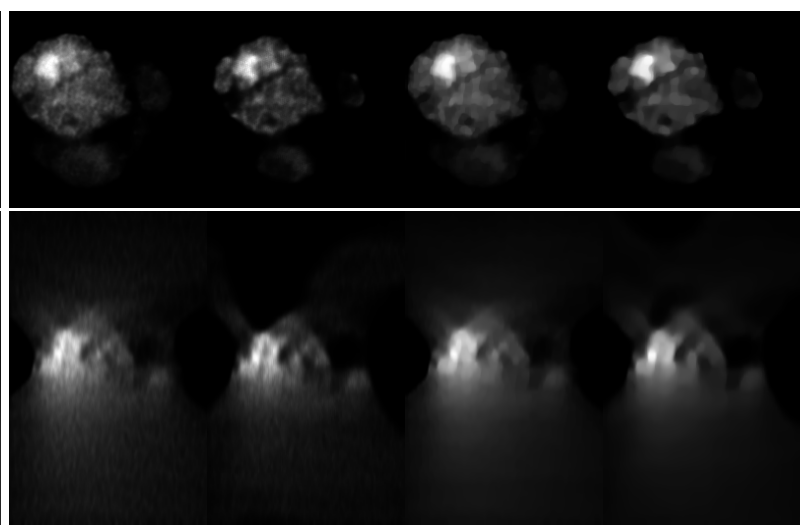

$\begin{array}{ll}\text { (c) RD+OTF mask (d) RD+KFTV } & \text { (e) TV+OTF mask (f) TV+KFTV }\end{array}$

$\begin{array}{lll}\lambda_{h}=5 & \lambda_{h}=5\end{array}$

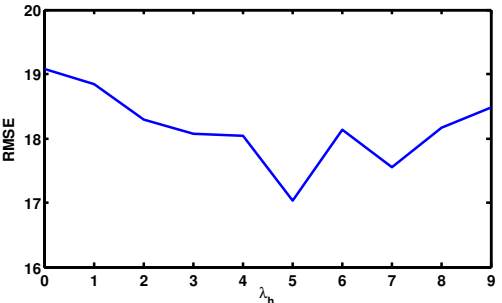

(g) RMSE depending on the chosen parameter $\lambda_{h}$ for KFTV with residual denoising $(\mathrm{RD})$ of the data.

Figure 9. Blind deconvolution results on a Drosophila S2 cell nucleus recording shown in the central xy-section (top row) and the yz-section indicated by the yellow line (bottom row). With KFTV, the superior blur reduction in z-direction is clearly visible.

TetraSpeck $0.2 \mu \mathrm{m}$ fluorescent point marker (bead) was recorded four times. The average of the bead recordings contains less noise than the single bead recordings and can therefore be used as initial PSF estimate.The identical Drosophila S2 cell nuclei were recorded with a spinning disk confocal microscope for comparison. The spinning disk recording was taken with a voxel size of $0.1 \times$ $0.1 \times 0.2 \mu \mathrm{m}^{3}$. Since the PSF of the spinning disk microscope is very small, this data can be used as pseudo ground truth to evaluate the deconvolution result from the widefield data. The recordings were registered with normalized cross-correlation. The whole dataset consists of 22 recorded nuclei that were cropped from five original recordings into separate volumes of about $100 \times 100 \times 100$ voxels before the deconvolution. The central slice and an xz-section of the spinning disk recording of one nucleus after registration is shown in figure 9(a), the same views of the widefield recording are displayed in figure 9(b). The results of our method KFTV with RD and TV regularization of the specimen function are shown in figure 9(c) - (f) for this nucleus. For the TV term, we choose $\lambda_{s}=0.001$. As expected, the results with the TV-regularized specimen function are smoother than the results with $\mathrm{RD}$, such that very fine structures in the central xy-plane are not restored. In terms of RMSE, the TV regularized OTF mask yields better results than the residual denoised OTF mask. With KFTV, residual denoising is sufficient to suppress noise in the reconstructed specimen function, yielding clearer structures in the reconstructed specimen and a lower RMSE.

To evaluate the influence of the parameter choice on the real dataset, we have computed the KFTV deconvolution with $\mathrm{RD}$ for different parameters $\lambda_{h}$ on the recording from 9(b). The resulting RMSE is given in figure $9(\mathrm{~g})$. The RMSE changes smoothly with the chosen parameter for $\lambda_{h} \leq 4$ and drops to a minimum at $\lambda_{h}=5$ for the example cell

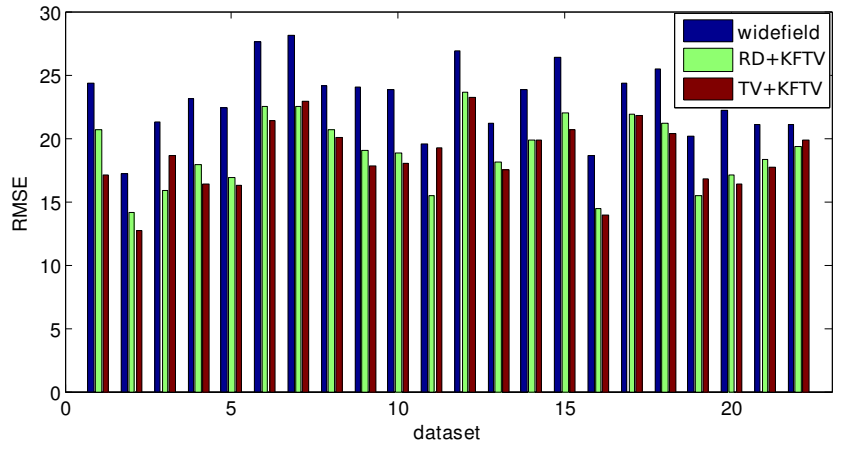

Figure 10. KFTV results on the 22 Drosophila S2 cell nucleus recordings in terms of RMSE.

nucleus. The resulting RMSE with $\lambda_{h}=4$ on the whole dataset of 22 nuclei with RD and TV regularization of the specimen function is given in figure 10. With KFTV and a TV-regularized specimen function, the average RMSE could be decreased from 23.02 to 18.58 , with RD to 18.89 .

\section{Conclusion}

We have presented a blind deconvolution method for widefield microscopic data that exploits the frequency domain properties of the widefield PSF. Accounting for the fact that the widefield OTF has a limited support and varies smoothly within this support, the presented KFTV method is based on a TV prior on the OTF values and a mask indicating the maximally possible OTF support. KFTV outperforms state-of-the-art deconvolution methods both visually and in terms of RMSE on two evaluation datasets, while it is independent from specific PSF simulation methods. On a new dataset of real widefield microscopic recordings showing Drosophila S2 cell nuclei, KFTV also shows convincing results. 
Acknowledgments This study was supported by the Excellence Initiative of the German Federal and State Governments (EXC 294) and ERC Starting Grant VideoLearn (279401).

\section{References}

[1] M. Born and E. Wolf. Principles of Optics: Electromagnetic Theory of Propagation, Interference and Diffraction of Light (7th Edition). Cambridge University Press, 1999. 2, 3

[2] R. W. Boyd. Radiometry and the detection of optical radiation. New York, John Wiley and Sons, 1983. 3

[3] A. Carasso. Linear and nonlinear image deblurring: A documented study. SIAM J. Numer. Anal., 36:1659-1689, 1999. 2,3

[4] J.-A. Conchello and J. McNally. Fast regularization technique for expectation maximization algorithm for optical sectioning microscopy. In C. J. Cogswell, G. S. Kino, and T. Wilson, editors, Three-Dimensional Microscopy: Image Acquisition and Processing III, volume 2655, pages 199208. Proc. SPIE, 1996. 2, 4

[5] N. Dey, L. Blanc-Feraud, C. Zimmer, P. Roux, Z. Kam, J. Olivo-Marin, and J. Zerubia. Richardson-Lucy algorithm with total variation regularization for 3D confocal microscope deconvolution. Microsc. Res. Techniq., 69:260-266, 2006. 2, 4

[6] S. Frisken-Gibson and F. Lanni. Experimental test of an analytical model of aberration in an oil-immersion objective lens used in three-dimensional light microscopy. J. Opt. Soc. Am. A, 9:154-166, 1992. 2, 3

[7] P. Green. Bayesian reconstructions from emission tomography data using a modified em algorithm. IEEE Trans. Med. Imag., pages 84-93, 1990. 4

[8] P. Green. On use of the em for penalized likelihood estimation. J. R. Stat. Soc. Series B, 52(3):443-452, 1990. 4

[9] M. Gustafsson, D. A. Agard, and J. W. Sedat. Sevenfold improvement of axial resolution in 3D widefield microscopy using two objective lenses. In Three-Dimensional Microscopy: Image Acquisition and Processing II, volume 2412, pages 147-156. Proc. SPIE, 1995. 3

[10] T. Holmes. Blind deconvolution of quantum-limited incoherent imagery: maximumlikelihood. J. Opt. Soc. Am. A, 9:1052-1061, 1992. 2, 4

[11] E. Hom, F. Marchis, T. Lee, S. Haase, D. Agard, and J. Sedat. Aida: an adaptive image deconvolution algorithm with application to multi-frame and three-dimensional data. J. Opt. Soc. Am. A, 24:1580-1600, 2007. 2, 6

[12] H. Ji and K. Wang. A two-stage approach to blind spatiallyvarying motion deblurring. In Computer Vision and Pattern Recognition (CVPR), pages 73-80, 2012. 2

[13] T. Kenig. Blind deconvolution in wide-field fluorescence microscopy, 2009. Master Thesis, Technion - Israel Institute of Technology, Haifa. 4, 6

[14] T. Kenig, Z. Kam, and A. Feuer. Blind image deconvolution using machine learning for three-dimensional microscopy. IEEE Trans. Pattern Anal. Mach. Intell., 32(12):2191-2204, 2010. 2, 4, 5, 6
[15] M. Keuper, M. Temerinac-Ott, J. Padeken, P. Heun, T. Brox, H. Burkhardt, and O. Ronneberger. Blind deconvolution with PSF regularization for widefield microscopy. In IEEE Int. Symp. on Biomedical Imaging (ISBI), pages 1292-1295, 2012. 5, 6

[16] H. Kirshner, D. Sage, and M. Unser. 3D PSF models for fluorescence microscopy in ImageJ. In $M A F^{\prime} 12$, page 154, 2011. 2, 3

[17] L. Lucy. An iterative technique for the rectification of observed distributions. The Astronomical Journal, 79/6:745754, 1974. 2

[18] J. Markham and J.-A. Conchello. Parametric blind deconvolution: a robust method for the simultaneous estimation of image and blur. J. Opt. Soc. Am. A, 16(10):2377-2391, 1999. 2

[19] J. Nuñez and J. Llacer. A fast bayesian reconstruction algorithm for emission tomography with entropy prior converging to feasible images. IEEE Trans. Med. Imag., 9/2:159171, 1990. 2

[20] P. Pankajakshan, L. Blanc-Feraud, Z. Kam, and J. Zerubia. Point-spread function retrieval for fluorescence microscopy. In IEEE Int. Symp. on Biomedical Imaging (ISBI), pages 1095-1098, 2009. 2

[21] J. Philip. Optical transfer function in three dimensions for a large numerical aperture. J. Mod. Optic., 46:1031-1042, 2009. 2, 3, 4

[22] W. Richardson. Bayesian-based iterative method of image restoration. J. Opt. Soc. Am. A, 62/1:55-59, 1972. 2

[23] P. Sarder and A. Nehorai. Deconvolution methods for 3-d fluorescence microscopy images. Signal Processing Magazine, 23(3):32-45, 2006. 1, 2, 6

[24] A. Schönle and S. Hell. Calculation of vectorial threedimensional transfer functions in large-angle focusing systems. J. Opt. Soc. Am. A, 19(10):2121-2126, 2002. 3

[25] P. Shaw. Comparison of widefield/deconvolution and confocal microscopy in three-dimensional imaging. In J. B. Pawley, editor, Handbook of Biological Confocal Microscopy. Springer, 1996. 2

[26] L. A. Shepp and Y. Vardi. Maximum likelihood reconstruction for emission tomography. IEEE Trans. Med. Imag., 1(2):113-122, 1982. 2

[27] C. Sheppard and M. Gu. Three-dimensional transfer functions for high- aperture systems. J. Opt. Soc. Am. A, 11:593598, 1994. 3

[28] J.-L. Starck, E. Pantin, and F. Murtagh. Deconvolution in astronomy: a review. Publ. Astron. Soc. Pac., 114:10511069, 2002. 4

[29] P. A. Stokseth. Properties of a defocused optical system. J. Opt. Soc. Am. A, 59:1314-1321, 1969. 2, 3, 6

[30] G. vanKempen, L. van Vliet, P. Verveer, and H. vanderVoort. A quantitative comparison of image restoration methods for confocal microscopy. J. Microsc., 185:354-365, 1997. 2, 4

[31] P. Verveer and T. Jovin. Efficient superresolution restoration algorithms using maximum a posteriori estimations with applicationto fluorescence microscopy. J. Opt. Soc. Am. A, 14:1696-1706, 1997. 4 